\title{
Production of the large scale superluminal ejections of the microquasar GRS 1915+105 by violent magnetic reconnection
}

\author{
E. M. de Gouveia Dal Pino ${ }^{1}$ and A. Lazarian ${ }^{2}$ \\ 1 Universidade de São Paulo, IAG, Rua do Matão 1226, Cidade Universitária, São Paulo 05508-900, Brazil \\ e-mail: dalpino@astro.iag.usp.br \\ 2 Department of Astronomy, University of Wisconsin, Madison, USA \\ e-mail: lazarian@astro.wisc.edu
}

Received 21 December 2004 / Accepted 13 June 2005

\section{ABSTRACT}

\begin{abstract}
We propose here that the large-scale superluminal ejections observed in the galactic microquasar GRS $1915+105$ during radio flare events are produced by violent magnetic reconnection episodes in the corona just above the inner edge of the magnetized accretion disk that surrounds the central $\sim 10 M_{\odot}$ black hole. The process occurs when a large-scale magnetic field is established by a turbulent dynamo in the inner disk region with a ratio between the gas+radiation and the magnetic pressures $\beta \simeq 1$, implying a magnetic field intensity of $\sim 7 \times 10^{8} \mathrm{G}$. During this process, substantial angular momentum is removed from the disk by the wind generated by the vertical magnetic flux therefore increasing the disk mass accretion to a value near (but below) the critical one $\left(\dot{M} \sim 10^{19} \mathrm{~g} \mathrm{~s}^{-1}\right)$. Part of the magnetic energy released by reconnection heats the coronal gas $\left(T_{\mathrm{c}} \lesssim 5 \times 10^{8} \mathrm{~K}\right)$ that produces a steep X-ray spectrum with luminosity $L_{\mathrm{X}} \simeq 10^{39} \mathrm{erg} \mathrm{s}^{-1}$, consistent with observations. The remaining magnetic energy released goes to accelerate the particles to relativistic velocities $\left(v \sim v_{\mathrm{A}} \sim c\right.$, where $v_{\mathrm{A}}$ is the Alfvén speed) in the reconnection site through first-order Fermi processes. In this context, two possible mechanisms have been examined that produce power-law electron distributions $N(E) \propto E^{-\alpha_{E}}$, with $\alpha_{E}=5 / 2,2$, and corresponding synchrotron radio power-law spectra with spectral indices which are compatible with that observed during the flares $\left(S_{v} \propto v^{-0.75,-0.5}\right)$.
\end{abstract}

Key words. acceleration of particles - accretion, accretion disks - black hole physics - magnetic fields

\section{Introduction}

Galactic and extragalactic accretion-powered sources often exhibit quasi-periodic variability and ejection phenomena that may offer important clues to the general physical processes in the inner regions of all classes of accreting sources, from young stars to X-ray binaries and AGNs.

The X-ray source GRS 1915+105 was the first galactic object to show evidence of superluminal radio ejection (Mirabel \& Rodriguez 1994, 1999) which is normally interpreted as due to relativistic jets. At a distance of $12.5 \mathrm{kpc}$ and probably with a 10 solar mass black hole in the center of a binary system, this microquasar offers an excellent laboratory for the investigation of black hole accretion and associated jet phenomena revealing, in time scales of minutes to months, a profusion of variable emission structures in a large range of wavelengths (Dhawan et al. 2000).

From a detailed analysis of the observed RXTE X-ray variability of this source, Belloni et al. (2000) have distinguished 12 different modes of variability that they interpreted as transitions between three basic states: a hard spectral-index state (denoted by C) with low X-ray luminosity, and two states (denoted by A and B) with softer spectra that were modeled as thermalized emission from an accretion disk, with state B being more luminous and hotter than state $\mathrm{A}$. They also found that after a long steady hard class, the most frequent class of variability was one characterized by a 30-min cycle.

Variability also has been observed in the radio and IR wavebands. Mirabel et al. (1998) reported simultaneous X-ray, IR and radio observations of these 30 -min cycles during which the X-ray emission is found to oscillate between states $\mathrm{A} / \mathrm{B}$ and C. The IR/radio emission of the plasma (which is consistent with synchrotron emission from an expanding nuclear jet; see below) seems to be associated with the times of recovery from the X-ray dips (see Figs. 2 and 3 from Mirabel et al. 1998), i.e., with the times the source is changing from a period in state $C$ to a period in which it rapidly oscillates between states $\mathrm{A} / \mathrm{B}$ and $\mathrm{C}$.

Large scale superluminal radio ejections have been also detected with MERLIN emerging from the source when this was at the end of a 3-week extended phase of low/hard X-ray state (also designated the plateau state; Fender et al. 1999; see also 
Fender et al. 2002). Similarly, in a compilation of (VLBA) radio and (RXTE and BATSE) X-ray data, taken during several weeks, Dhawan et al. (2000, hereafter DMR) have distinguished two main states of the system, a plateau and a flare state. The plateau state is characterized by a flat radio spectrum $\left(S_{v} \propto v^{0}\right)$ coming from a compact region of size of a few $\mathrm{AU}$, and flux density 1-100 mJy. During this phase, the associated RXTE (2-12 keV) soft X-ray emission is weak, while the BATSE (20-100 keV) hard X-rays are strong. On the other hand, during the flare phase, optically thin ejecta are superluminally expelled up to thousands of AU with fluxes up to $1 \mathrm{Jy}$ at $\lambda 13 \mathrm{~cm}\left(S_{v} \propto v^{-0.6}\right)$ with rise time $\lesssim 1$ day and which fade after several days. The soft $\mathrm{X}$-rays also flare during this phase and exhibit high variability, while the hard X-rays fade for a few days before recovering (see Figs. 1 and 4 of DMR). The radio imaging over a range of wavelengths $(13,3.6,2.0$, and $0.7 \mathrm{~cm}$ ) resolves the nucleus as a compact jet of length $\sim 10 \lambda_{\mathrm{cm}}$ AU. This nuclear jet varies on time scales of $\sim 30$ min during minor X-ray/radio bursts and reestablishes within $\sim 18 \mathrm{~h}$ after a major outburst. So far, GRS $1915+105$ has been the only system among the galactic accreting black holes of stellar mass where both AU-scale steady jets and large scale superluminal ejections have been unambiguously observed.

It is generally believed that the X-ray emission of galactic black holes and active galactic nuclei arises from hot gas accretion flow or from an accretion-disk corona. In the first class of models, advection-dominated accretion flow (ADAF) of disk material into the black hole (e.g., Narayan et al. 1997), as well as simultaneous advection-dominated inflow-outflow (ADIOS) solutions (Blandford \& Begelman 1999) have been proposed. In the second class, the presence of magnetic fields adds complexity to the solutions but plays an essential role in the disk-coronal coupling, in the energy and angular momentum transport and in jet production and acceleration (e.g., Liu et al. 2002).

The AU-scale nuclear jet of GRS 1915+105 developed in the plateau phase has been interpreted as synchrotron emission from relativistic electrons (e.g., Falke \& Biermann 1999), and its origin has been attributed to disk instabilities in an ADAF-type flow by Belloni et al. (1997a, b). According to this model, the 30-min soft X-ray variability observed during the plateau state, which involves a transition between states $\mathrm{C}$ and $\mathrm{A} / \mathrm{B}$, is explained in terms of periodic evacuation and refilling of the inner disk region on time scales of seconds as a result of thermal viscosity. This process is believed to occur in the region where the radiation pressure dominates the gas pressure and the time required to refill the disk with X-ray emitting hot gas is set by the thermal viscous time scale. The observation of radio oscillations with similar period intervals supports the idea that the synchrotron emission of the nuclear jet is fed by relativistic plasma injected from the inner disk during the dips in the soft X-ray flux (DMR).

In contrast, the large superluminal radio flares observed at the $\sim 500 \mathrm{AU}$ scales cannot be explained by the same viscous disk instability model, because they eject an order of magnitude more mass than the AU-scale mini-jet and the recovery time scale (of $\sim 18 \mathrm{~h}$ ) would require according to that model a much larger evacuation radius of disk material well beyond the radiation pressure-dominated region where the instability occurs.

Recently, different groups have proposed alternative scenarios to explain the variability of the GRS 1915+105 microquasar. Livio et al. (2003; see also King et al. 2004) have suggested that the inner region of the accretion disk would switch between two states. In one of them (state A/B), the accretion energy would be dissipated locally to produce the observed disk luminosity, and in the other (state $\mathrm{C}$ ) the accretion energy would be converted into magnetic energy and emitted in the form of a relativistic jet (state C). They have attributed the transition between the two states to dynamo generation of a global poloidal magnetic field. Similarly, Tagger et al. (2004), also focusing mainly on the 30-min cycles, have suggested that the alternations between the high-soft and the low-hard states and the production of a nuclear relativistic jet could be controlled by the successive accumulation and release of poloidal magnetic flux (e.g., by magnetic reconnection) in the innermost parts of the accretion disk. They have proposed a specific model to explain the quasi periodic variability in the disk emission based on the development of the accretion-ejection instability (Tagger \& Pellat 1999). This is a global mode formed by spiral waves that travel back and forth in the inner regions of a disk threaded by a poloidal magnetic field with a ratio $\beta$ between the gas pressure and the magnetic pressure close to unity. The spiral waves become unstable by extracting energy and angular momentum from the inner region (thus increasing the accretion) and transferring it outward.

de Gouveia Dal Pino \& Lazarian (2004) have suggested the possibility that the superluminal large scale radio flares of GRS $1915+105$ could be produced by relativistic plasma accelerated during violent magnetic reconnection events occurring in the magnetized corona just above the inner regions of the accretion disk. In the present work, we try to show that violent magnetic reconnection could indeed occur in the innermost parts of the disk and produce the large scale superluminal ejections when $\beta$ approaches one and the mass accretion energy becomes close to the critical value.

In Sect. 2 we draw the potential scenario for flare production and the superluminal ejecta. In Sect. 3, we try to quantify it by computing the disk and coronal parameters and evaluating the total amount of magnetic energy released during violent reconnection in the corona; in Sect. 4, we evaluate both the electron and the synchrotron power-law spectra that are produced by the accelerated particles through first-order Fermi process in the reconnection site. We conclude (in Sect. 5) with a brief discussion of the implications of our results.

\section{A simple possible scenario}

\subsection{The $\mathrm{BH}$ magnetosphere}

Magnetized accretion disks around rotating (Kerr) black holes (BHs) are frequently invoked to explain the high energy radiation and jet production and collimation in quasars and microquasars. The magnetic field lines originally frozen in the disk plasma will deposit along with accreting gas onto the $\mathrm{BH}$ horizon, therefore developing a magnetosphere around the horizon 
(see, e.g., Wang et al. 2002). Near the horizon, no matter how chaotic the field threading the disk, the field through the hole will become ordered. If the disk tries to deposit a chaotic field on the hole, the field's closed loops will destroy themselves on a time scale $t \simeq R_{\mathrm{H}} / c \sim 10^{-4} \mathrm{~s} M_{10}$, leaving the field ordered. If on the other hand, magnetic pressure from field lines threading the disk temporarily push the hole's field into a clumped configuration, it will spring back on this same time scale and make itself more uniform (MacDonald et al. 1986).

The magnetic coupling (MC) of a rotating black hole with its surrounding disk has been investigated in some detail (see e.g., Blandford 1999; Wang, Lei \& Ma 2003, and references therein). The presence of some closed field lines connecting the $\mathrm{BH}$ with the inner disk edge (in a variant of the Blandford-Znajek process 1977), can lead to energy and angular momentum transfer between the $\mathrm{BH}$ and the disk if they are rotating at different angular speeds. Though not a necessary condition, we will assume, for simplicity, in the present study that the $\mathrm{BH}$ and the inner disk edge are nearly co-rotating so that no significant angular momentum and energy transfer is occurring between them. The magnetic structure near the $\mathrm{BH}$ horizon will have a force-free configuration determined by the MC process and the strength of the field near the hole will be determined by the disk's past history. The net flux today must be equal to the total flux that the disk has deposited in its entire past lifetime. These deposits will cause the net flux through the hole to fluctuate stochastically. The rms field will grow with time until it becomes so strong that it pushes back into the accretion disk and slows down accretion. The resulting maximum strength is likely to be such as to produce equilibrium between gas ram pressure and magnetic pressure in the innermost parts of the disk (Wang et al. 2003), though Parker-Rayleigh-Taylor instabilities may reduce this strength (MacDonald et al. 1986).

\subsection{The accretion disk and corona around the $B H$}

In the standard model for an accretion disk around a black hole, gravitational energy is continually converted into kinetic, thermal and magnetic energy by the joint action of viscosity and magnetic field line stretching and reconnection. The viscosity can be mainly turbulent or magnetic or both (Shakura-Sunyaev 1973).

In the process of angular momentum transport in the disks, shear is expected to convert an existing poloidal magnetic field into a toroidal component. Then, probably some of the toroidal flux will be used to generate new poloidal fields by raising magnetic loops in a dynamo process. Most of the energy will be either dissipated within the disk that will then emit thermalized radiation, or in the low density corona above and below the disk that will radiate thermally or by Compton scattering, or else the energy will be released in the form of a bulk flow, as a jet. In the last case, it is possible that essentially all the accretion energy of the inner disk is lost in the jet (see, Blandford \& Payne 1982; Livio et al. 2003, and references therein).
Let us assume as in previous works (e.g., Merloni 2003; Livio et al. 2003; Tagger et al. 2004; de Gouveia Dal Pino \& Lazarian 2004) that the inner parts of the accretion disk alternate between two states which are controlled by changes in the global magnetic field. Most of the magnetic field generated in a dynamo process in which the turbulence is driven by, e.g., the magneto-rotational instability (Balbus \& Hawley 1991), is a tangled small-scale magnetic field within the disk. As remarked by Livio et al. (2003), as long as the magnetic field remains as such, most of the dissipation will occur locally within the disk that will be radiative and very luminous. However, if a large scale poloidal magnetic field is generated then it is possible that an energetic outflow can be driven.

Numerical simulations of the dynamo process in accretion disks (e.g., Kudoh et al. 2002; Shibata 2003) are still unable to compute the generation of a global poloidal magnetic field, so that for the time being we must rely on physical estimations rather than on detailed modeling (Livio et al. 2003). Tagger et al. (2004), for example, have suggested that a gradual increase in the magnetic flux in the inner regions of the accretion disk could be achieved by the combined action of the turbulent dynamo associated, e.g., with the magneto-rotational instability (which would create a vertical flux of opposite polarities in the inner and outer disk regions), followed by advection of the magnetic flux with the gas to the inner disk regions. Although magnetic diffusivity could interrupt this magnetic flux accumulation, there is at least some observational evidence for it in the case of the magnetic field structure of our own galactic disk that shows a strong vertical field in the inner tens of pcs of the galactic center which is almost three orders of magnitude more intense than the horizontal magnetic fields measured elsewhere in the disk. Livio (1997; see also Livio et al. 2003; King et al. 2004), in addition, assuming a turbulent dynamo process with inverse cascade and reconnection of magnetic loops above and below the disk, have demonstrated that (provided that the distribution $n(l)$ of the lengths of the magnetic loops satisfies the relation $n(l) \propto l^{-3 / 2}$ ) large scale poloidal fields can be generated such that $L_{\mathrm{j}} \sim L_{\mathrm{acc}}$, where $L_{\mathrm{j}}$ is the jet power and $L_{\mathrm{acc}}$ is the disk accretion luminosity.

Considering the aspects above, we assume here that during the plateau state that precedes a radio flare, a large scale poloidal field is progressively built in the disk by a turbulent dynamo process. The action of buoyancy forces will also make the disk unstable against the Parker-Rayleigh-Taylor instability and horizontal magnetic field lines will raise from the disk forming large scale loops in the rarified hot corona. We further assume that once the dynamo process establishes a global poloidal field over a substantial region of the disk, it will be able to maintain that field for a period of time. The length of time the source spends in this state is hard to estimate without a complete physical model, however observations indicate that the system remains in the plateau state for prolonged periods of time.

The vertical field flux will give rise to a wind that will remove angular momentum from the disk, therefore significantly increasing the accretion rate (possibly at a rate greater than the rate due to the disk viscosity). Also, with the accumulation (trap) of vertical flux in the inner regions the ratio between the 


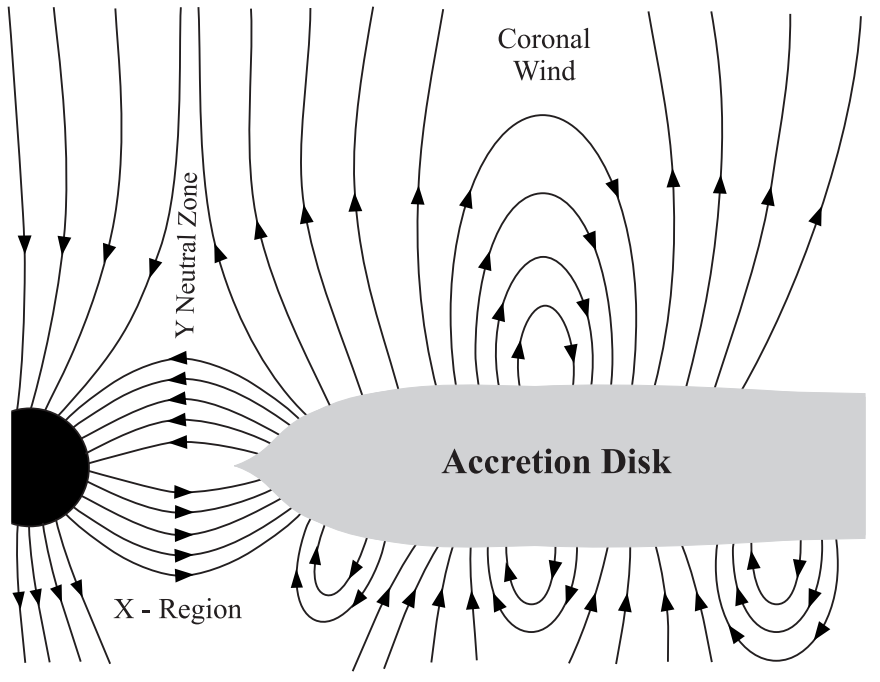

Fig. 1. Schematic drawing of the magnetic field geometry in the inner disk region at $R_{\mathrm{X}}$. The acceleration occurs in the magnetic reconnection site at the $\mathrm{Y}$ type neutral zone (see the text).

gas+radiation pressure to the magnetic field pressure $(\beta)$ will soon decrease to one. Under these conditions, events of reconnection of magnetic field lines with opposite polarization will be inevitable, and in the innermost regions this process may become eventually very violent when enough magnetic energy is stored in the corona. We show below that this occurs when $\beta \sim 1$ and the accretion rate approaches the critical value. We then argue that this could explain the large scale radio flares in GRS 1915+105 with the ejection of the observed relativistic blobs from the innermost regions.

\subsection{Magnetic reconnection site in the inner disk-corona region}

Considering the assumptions above, the resulting qualitative structure of the magnetosphere of the hole and the accretion disk must be like that shown in Fig. 1 (which was adapted from MacDonald \& Thorne 1982; and MacDonald et al. 1986). In the inner disk region (of radius $R_{\mathrm{X}}$ ), there is a site which may be appropriate for violent magnetic reconnection. Surfaces of null poloidal field lines mediate the geometry of the open field lines anchored into the $\mathrm{BH}$ horizon with the opened lines of the disk wind and those connecting the disk with the $\mathrm{BH}$ horizon. Labeled as "Y neutral zone" in Fig. 1, these magnetic null surfaces begin or end on Y points. Across each null surface, the poloidal field suffers a sharp reversal of direction. According to Ampère's law, large electric currents must flow out of the plane shown in Fig. 1, along the null surfaces, and in the presence of finite electric resistivity, dissipation of these currents will lead to reconnection of the oppositely-directed field lines (e.g., Biskamp 1997; Lazarian \& Vishniac 1999). We here investigate the possibility that the magnetic energy released by reconnection near the Y point region (see also Fig. 2) is able to accelerate the plasma to relativistic velocities and produce the synchrotron radio flares (see below).

The release of magnetic energy by reconnection is believed to be the most promising mechanism for production of solar

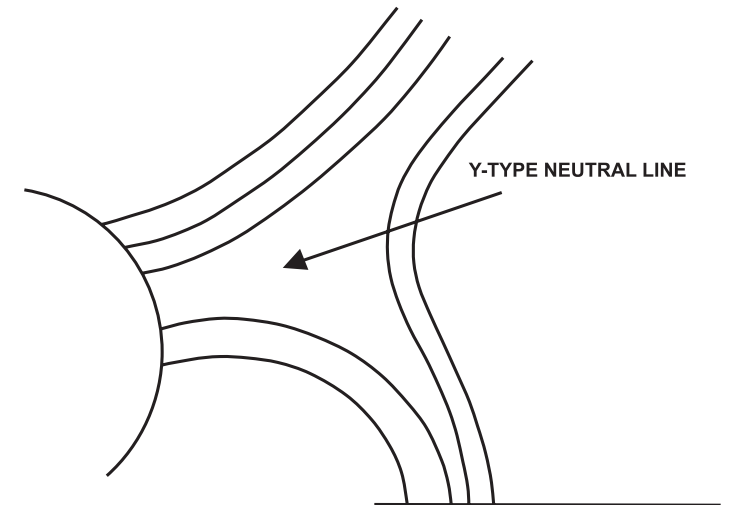

Fig. 2. A closer view of the Y-type neutral zone where the field lines coming from the central source encounter those opened by the disk wind and those trapped by the funnel inflow emanating from the $R_{\mathrm{X}}$ region to form a Y point of null magnetic field.

flares. Both direct and indirect astrophysical evidence show that fast reconnection occurs in spite of the theoretical difficulties involved (see, e.g., Lazarian et al. 2004). Y-type neutral zones are also present, for example, in eruptive magnetic arcades of the solar corona, like those associated with two-ribbon solar flares (see, e.g., Takasaki et al. 2004; Sakajiri et al. 2004). In these cases, a possible interpretation of the observations is that a current sheet forms between an emerging magnetic flux and the pre-existing magnetic flux of opposite polarization and magnetic reconnection in this zone will cause the formation of a filament/plasmoid. This will temporally inhibit further reconnection and establish a temporally stable magnetic configuration. During this stage, there will be considerable magnetic energy storage due to the continuing converging motion of the two fluxes. Eventually an eruption develops (for example, from sheared or twisted arcades lying underneath the magnetic null point), the filament/plasmoid is then ejected and a large quantity of magnetic energy is released (see, e.g., Takasaki et al. 2004).

Lazarian \& Vishniac (1999) proposed a mechanism by which flares can naturally emerge as a result of an instability mediated by turbulence. In their model the reconnection rate increases due to magnetic field wandering caused by turbulence. Fluxes of non-parallel magnetic field lines reconnect very slowly if the turbulent velocities are much smaller than the Alfven velocity. However, outflows caused by reconnection increase the level of turbulence and therefore the reconnection rate. As a result of such a positive feedback a flare develops.

Whatever is the particular mechanism, the energy released by magnetic reconnection is expected to ultimately drive violent outward motion in the surrounding plasma that can produce copious amounts of solar cosmic rays via direct acceleration along the open magnetic field lines or via a first order Fermi process (e.g., Reames 1995). Similar mechanisms have been recently proposed to accelerate the plasma in magnetic reconnection zones of young accretion-induced collapse pulsars to produce ultra-high energy cosmic rays (de Gouveia Dal Pino \& Lazarian 2000, 2001).

The several stages described above for magnetic reconnection and solar flare production may also be operating in 
our accretion disk-corona-BH system, but since in the present study it is not crucial to follow the detailed evolution of the magnetic configuration and of the features that develop during the reconnection process, we will consider a simpler scenario. According to the discussion in the previous sections, we argue that whenever $\beta$ becomes small enough and the accretion rate attains values near the critical one in the inner disk region, then the lines of opposite polarization near the Y-zone will be pressed together by the accreted material sufficiently rapidly as to undergo violent reconnection events. Energy stored in the magnetic field will be released suddenly to heat the plasma and accelerate charged particles. Some of the high speed particles will travel downward to the $\mathrm{BH}$ or to the disk to the foot points of the magnetic-arches, yielding bright X-ray emission, others will spew outward giving rise to the relativistic radio ejecta (see below) ${ }^{1}$.

We notice that the merging of the closed field lines of the loops and the open field lines in the disk further out from the inner radius can also lead to reconnection, however, we find that the amount of magnetic energy released in these regions is not sufficient to accelerate the particles to produce the observed radio luminosities in the large flares (see below).

For reconnection to occur in the inner disk region $R_{\mathrm{X}}$, near the $\mathrm{Y}$ point, the poloidal flux from the disk and the flux anchored in the central magnetic structure surrounding the BH must have opposite signs, as in Fig. 1. As pointed out by Tagger et al. (2004), this suggests that large ejections with violent flares will occur only when the system is in that configuration. Violent reconnection will not take place in the periods when both fluxes have the same sign. This dichotomy is observed for example in the interaction between the terrestrial and the solar wind magnetic field. Now, the magnetic flux trapped in the BH horizon is a sum of all magnetic flux accreted during the whole history of the source. If the magnetic flux in the disk is either produced by a turbulent dynamo in the disk itself or advected from a companion star, its sign is expected to change from time to time, although the characteristic time scale of this variation is difficult to compute in the absence of a detailed modelling of the dynamo in the disk or in the companion star. In the long term, a sufficient number of reconnection events might in turn cancel the flux trapped in the BH horizon and change its sign as well. In the case of the solar dynamo, it is not clear yet why the field reversals take place every 22 years. In the case of GRO 1915+105, Tagger et al. (2004) have suggested that this long term evolution of the field configuration could be of the order of one to a few years so that part of the time the field fluxes in the inner disk edge and in the hole would be antiparallel and the rest of the time parallel.

As remarked above, observations of the Sun indicate that the amplification, dissipation and reconnection of the magnetic field lines is likely to occur not steadily but sporadically,

\footnotetext{
${ }^{1}$ In the scenario just described, the high accretion rate may also cause the compression and shear of magnetic loops that may exist in the lower parts of the inner disk corona, under the Y-zone dome. These could also undergo violent reconnection and even produce plasmoids that could eventually erupt and provoke particle acceleration as well, as in the Sun (see discussion in Sect. 4).
}

involving violent flares followed by more gradual recoveries to the pre-flare magnetic field configuration with no obvious periodicity. A similar behavior, i.e., violent flares followed by more slowly recovering of the pre-flare state is also detected in the observed superluminal radio ejecta of GRS $1915+105$, thus suggesting that magnetic reconnection could be also associated to the production of relativistic blob ejections that rapidly move to the $\sim 100 \mathrm{AU}$ scales. In the next section, we try to quantify the ideas just described.

We notice that after reconnection, the partial destruction of the magnetic flux in the inner disk will make $\beta$ increase and make the disk return to the less magnetized condition of the plateau state with most of the energy being dissipated locally within the disk instead of in the outflow.

\section{Building the model for the superluminal ejections during the flares}

We adopt the field geometry of the magnetized accretion disk/corona as described in the previous section (Fig. 1).

We first make an order-of-magnitude estimate of the amount of magnetic energy that could be extracted from the inner disk region by simply assuming that it is approximately equal to the disk accretion energy rate $G M_{\star} \dot{M} / R_{\mathrm{X}}$. For a $\mathrm{BH}$ with mass $M_{\star}=10 M_{\odot}=M_{10}$ and Schwartzschild radius $R_{\star}=2 G M_{\star} / c^{2}=2.96 \times 10^{6} M_{10} \mathrm{~cm}$, this gives

$\dot{W}_{B} \simeq 10^{39} \mathrm{erg} \mathrm{s}^{-1} M_{10} \dot{M}_{19} R_{\mathrm{X}, 7}^{-1}$

where we have assumed that the inner radius of the accretion disk $\left(R_{\mathrm{X}}\right)$ corresponds approximately to the last stable orbit of the $\mathrm{BH}, R_{\mathrm{X}} \simeq 3 R_{\star} \simeq 10^{7} \mathrm{~cm}$, and that the accretion rate $\dot{M} \lesssim \dot{M}_{\text {Edd }}$, where $\dot{M}_{\text {Edd }}=1.9 \times 10^{19} M_{10} \mathrm{~g} \mathrm{~s}^{-1}$ is the Eddington critical accretion rate (Shakura \& Sunyaev 1973), $\dot{M}_{19}$ is $\dot{M}$ in units of $10^{19} \mathrm{~g} \mathrm{~s}^{-1}$, and $R_{\mathrm{X}, 7}$ is $R_{\mathrm{X}}$ in units of $10^{7} \mathrm{~cm}$. This estimate of $\dot{W}_{B}$ can be compared to the observed X-ray luminosity of GRS $1915+105\left(\sim 10^{39} \mathrm{erg} \mathrm{s}^{-1}\right)$.

Now, in order to evaluate more precisely the amount of magnetic energy that can be extracted through violent reconnection from the $Y$ neutral zone to produce superluminal ejecta, we need first to evaluate the physical conditions both in the magnetized disk and corona.

\subsection{Disk and coronal parameters}

To evaluate the disk quantities at $R_{\mathrm{X}}$, we employ the standard disk model (Shakura \& Sunyaev 1973; Frank et al. 1992). In the inner disk region (named the X-region), the radiation pressure is larger than the thermal pressure and the disk will be radiation pressure-dominated. In this case,

$T_{\mathrm{d}} \simeq 1.2 \times 10^{8} \mathrm{~K} \alpha_{0.1}^{-1 / 4} M_{10}^{1 / 4} R_{\mathrm{X}, 7}^{-3 / 4}$

and

$n_{\mathrm{d}} \simeq 2.8 \times 10^{17} \mathrm{~cm}^{-3} \alpha_{0.1}^{-1} M_{10}^{-1 / 2} \dot{M}_{19}^{-2} R_{\mathrm{X}, 7}^{3 / 2} q_{0.82}^{-8}$

where $q=\left[1-\left(R_{\star} / R_{\mathrm{X}}\right)^{1 / 2}\right]^{1 / 4}=0.82, T_{\mathrm{d}}$ and $n_{\mathrm{d}}$ are the disk gas number density and temperature, respectively, $\alpha_{0.1}$ is the Shakura-Sunyaev viscous coefficient in units of 0.1 (e.g., 
Frank et al. 1992), and $\dot{M}_{19}$ is the disk accretion rate in units of $10^{19} \mathrm{~g} \mathrm{~s}^{-1}$.

The width $H$ of the disk in the radiation pressure-dominated region is given by

$\frac{H}{R_{\mathrm{X}}} \simeq 0.7 \dot{M}_{19} R_{\mathrm{X}, 7}^{-1} q_{0.82}^{4}$.

As remarked in the previous section, the violent magnetic reconnection events that may generate large scale relativistic ejections are expected to occur when the large scale magnetic field in the inner disk region, which is generated by dynamo process, attains an intensity as large as $B_{\mathrm{d}}^{2} / 8 \pi \simeq P_{\mathrm{d}}$, where $P_{\mathrm{d}}$ is the disk pressure which is dominated by the radiation pressure, i.e., when $\beta \simeq 1$, giving

$B_{\mathrm{d}} \simeq 6.9 \times 10^{8} \mathrm{G} \alpha_{0.1}^{-1 / 2} M_{10}^{1 / 4} R_{\mathrm{X}, 7}^{-3 / 4}$.

Liu et al. (2002) proposed a simple model to quantify the parameters of a magnetized disk-corona system. Assuming, as in the solar corona, that gas evaporation at the foot point of a magnetic flux tube quickly builds up the density of the corona to a certain value, and the tube radiates away the heating due to magnetic reconnection through Compton scattering, they derived the following coronal quantities (as a function of disk quantities) for a radiation pressure-dominated disk

$n_{\mathrm{c}} \simeq 7.6 \times 10^{14} \mathrm{~cm}^{-3} \alpha_{0.1}^{-15 / 16} \beta_{1}^{-3 / 4} M_{10}^{-25 / 32} \dot{M}_{19}^{-2} R_{\mathrm{X}, 7}^{75 / 32} l_{8}^{-3 / 4}$

and

$T_{\mathrm{c}} \simeq 4.9 \times 10^{8} \mathrm{~K} \alpha_{0.1}^{-15 / 32} \beta_{1}^{-3 / 8} M_{10}^{-25 / 64} \dot{M}_{19}^{-1} R_{\mathrm{X}, 7}^{75 / 64} l_{8}^{1 / 8}$

where $n_{\mathrm{c}}$ and $T_{\mathrm{c}}$ are the coronal gas number density and temperature, respectively, $\beta_{1}$ is the value of $\beta$ in the disk in units of 1 , and $l_{8}$ is the scale height of the Y neutral zone in the corona in units of $10^{8} \mathrm{~cm}$. Although the quantities above have been computed for the purposes of the present model only in the inner disk radius $R_{\mathrm{X}}$, they are valid for any radius of the disk-corona within the radiation pressure-dominated region. According to Liu et al.'s model, smaller values of $\beta$ would result in more energy dissipation through the corona and higher coronal temperature and density, as indicated by Eqs. (6) and (7).

\subsection{Rate of magnetic energy release in the Y-type neutral zone}

Let us assume that the poloidal magnetic field that rises in the corona just above the inner disk region $\left(B_{\mathrm{X}}\right)$ is of the order of the local disk magnetic field, $B_{\mathrm{d}}$ (Eq. (5)), that has been produced and pushed to the inner disk region as described in the previous section (see also, e.g., Arons 1993; de Gouveia Dal Pino \& Lazarian 2001)².

The rate of magnetic energy that can be extracted from the Y-zone in the corona (above and below the disk) through reconnection is $\dot{W}_{B} \simeq\left(B_{\mathrm{X}}^{2} / 8 \pi\right) \xi v_{\mathrm{A}}\left(4 \pi R_{\mathrm{X}}\right) L_{\mathrm{X}}$, where $\xi=v_{\text {rec }} / v_{\mathrm{A}}$ is

\footnotetext{
${ }^{2}$ We notice that this value is a little larger than the one estimated by assuming, e.g., that the ejected gas takes the bulk of the accretion power, i.e., $L_{\mathrm{j}} \sim L_{\text {acc }}$ (where $L_{\mathrm{j}} \simeq B_{\mathrm{c}}^{2} R_{\mathrm{X}}^{2} v_{\phi} / 2, L_{\mathrm{acc}} \simeq \dot{M} v_{\phi}^{2}$, and $v_{\phi}$ is the Keplerian toroidal velocity in the disk; see also Livio et al. 2003).
}

the reconnection efficiency factor, $v_{\text {rec }}$ is the reconnection velocity, $v_{\mathrm{A}}=B_{\mathrm{X}} /\left(4 \pi n_{\mathrm{c}} m_{\mathrm{p}}\right)^{1 / 2}$ is the coronal Alfvén speed, $m_{\mathrm{p}}$ is the hydrogen mass, and $L_{\mathrm{X}}$ is the length of the reconnection region which according to Fig. 3 is $L_{\mathrm{X}} / \Delta R_{\mathrm{X}} \approx v_{\mathrm{A}} / \xi v_{\mathrm{A}} \approx \xi^{-1}$. For the conditions investigated here we find that $v_{\mathrm{A}} \simeq c$. Substitution of these relations into the equation above gives

$\dot{W}_{B} \simeq \frac{B_{\mathrm{X}}^{2}}{8 \pi} c\left(4 \pi R_{\mathrm{X}}\right) \Delta R_{\mathrm{X}}$

In order to estimate $\Delta R_{\mathrm{X}}$ (or $L_{\mathrm{X}}$ ), we need to model the reconnection process itself. Classical reconnection schemes provide too slow reconnection rates. However, there is ample observational evidence that reconnection may take place at very high rates which are comparable with the Alfvén speed (see e.g., Dere 1996). In the framework of solar flares, considerable progress has been made in recent years towards the determination of more realistic reconnection rates during flare events based mostly on the separation speed of $\mathrm{H} \alpha$ flare ribbons (e.g., Qiu et al. 2004; Jing et al. 2005; Sakajiri et al. 2004; Takasaki et al. 2004). These observations indicate that the reconnection speed can be as high as few tenths of the Alfvén velocity (Takasaki et al. 2004). Since in the conditions we deal with in the present analysis this speed approaches $c$, the corresponding reconnection rate can be large.

As mentioned earlier, in the turbulent reconnection model proposed by Lazarian \& Vishniac (1999, henceforth LV99, 2000; see also Lazarian 2005, for an extensive review on this matter) wandering of magnetic field lines was suggested as the cause of fast reconnection. An alternative model (Bisckamp et al. 1997; Shay et al. 2003) attempts to explain fast reconnection by plasma properties, such as anomalous resistivity (Parker 1979). These models do not exclude each other. For instance, while dealing with anomalous resistivity in most astrophysical situations, one has also to deal with the effects of turbulence. At the same time the reconnection rates in the LV99 model may get an initial boost from the anomalous resistive effects. This boost may be important when the initial turbulent velocities are much smaller than the Alfven speed. In our present analysis, we focus our attention on regions of strong magnetic fields, like those around the $\mathrm{BH}$ in the $\mathrm{X}$-region during nearly critical accretion. For those regions the resistivity should be anomalous and the fast reconnection should occur (with $v_{\text {rec }} \sim v_{\mathrm{A}}$ ).

Following LV99, we can estimate the width of the current sheet for which the resistivity should be anomalous:

$\Delta R_{\mathrm{X}}=\frac{c \Delta B}{4 \pi n_{\mathrm{c}} Z e v_{\mathrm{th}, \mathrm{c}}}$

where $\Delta B \simeq 2 B_{\mathrm{X}}$ denotes the change of the magnetic field across the reconnection region, and $v_{\mathrm{th}, \mathrm{c}}=\left(\frac{5 / 3 k T_{\mathrm{c}}}{m_{\mathrm{p}}}\right)^{1 / 2}$ the thermal velocity of the ions of charge $Z e$ in the corona

$v_{\mathrm{th}, \mathrm{c}}=2.65$

$$
\times 10^{8} \mathrm{~cm} \mathrm{~s}^{-1} \alpha_{0.1}^{-15 / 64} \beta_{1}^{-3 / 16} M_{10}^{-25 / 128} \dot{M}_{19}^{-1 / 2} R_{\mathrm{X}, 7}^{75 / 128} l_{8}^{1 / 16} .
$$

Substitution of Eqs. (5), (6), and (10) into Eq. (9) results

$\left(\frac{\Delta R_{\mathrm{X}}}{R_{\mathrm{X}}}\right) \simeq$

$$
0.0034 Z^{-1} \alpha_{0.1}^{43 / 64} \beta_{1}^{15 / 16} M_{10}^{157 / 128} \dot{M}_{19}^{5 / 2} R_{\mathrm{X}, 7}^{-599 / 128} l_{8}^{11 / 16} .
$$




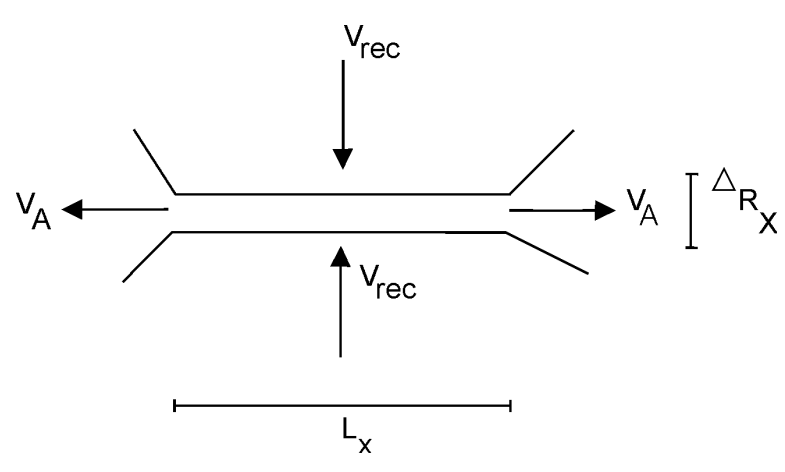

Fig. 3. Schematic representation of a reconnection region.

Now, if we plug the result above and Eqs. (5) into (8), we get

$\dot{W}_{B} \simeq 2.4 \times 10^{39} \mathrm{erg} \mathrm{s}^{-1} \alpha_{0.1}^{-1} M_{10}^{1 / 2} R_{\mathrm{X}, 7}^{1 / 2}\left(\frac{\Delta R_{\mathrm{X}} / R_{\mathrm{X}}}{0.0034}\right)$

and the corresponding reconnection time is

$t_{\mathrm{rec}} \simeq \frac{R_{\mathrm{X}}}{\xi v_{\mathrm{A}}} \simeq 3.3 \times 10^{-4} \mathrm{~s}^{-1} \xi^{-1} R_{\mathrm{X}, 7}$

which indicates that the release of magnetic energy is very fast.

The analysis of Eqs. (11) and (12) shows that the adoption of a somewhat smaller viscosity parameter $\alpha$ (which would imply a more quiescent disk), by an order of magnitude, for instance, would require a disk accretion rate only a few times larger in order to keep the values of $\Delta R_{\mathrm{X}} / R_{\mathrm{X}}$ and $\dot{W}_{B}$ unaltered.

Equation (12) gives the total expected amount of magnetic energy released by fast reconnection in the Y-zone during the flare of GRS 1915+105. Part of this energy will heat the gas that will produce X-ray luminosity, and the remaining will accelerate the charged particles to relativistic velocities producing a violent radio ejecta. But what is the fraction that goes to each part, and what is the potential acceleration mechanism in the reconnection site that can produce the superluminal radio ejections?

The evaluation of the exact fraction of the magnetic energy that goes to each of the processes above is a task beyond of the scope of the present work, as it depends on the detailed modelling of the conditions of the disk-corona and of the efficiency of each process. However, assuming that most of the magnetic energy released through reconnection in the corona goes to heat the plasma, Liu et al. (2002) have computed the average value of the Compton parameter $(y)$ for an optically thin corona with an original distribution of thermal electrons, $y=\frac{4 k T_{\mathrm{c}}}{m_{\mathrm{e}} c^{2}} n_{\mathrm{c}} \sigma_{\mathrm{T}} l$, which was weighted over all the disk radii. According to their results, for a disk near the critical accretion $\left(\dot{M} \sim \dot{M}_{\text {Edd }}\right)$, $y<0.8$, which implies a soft (and steep) X-ray spectrum, as required by GRS 1915+105 observations during the flare state, with a spectral index $\alpha_{r-x}=\left(\frac{9}{4}+\frac{4}{y}\right)^{1 / 2}-\frac{3}{2}>1.2$. This result is qualitatively consistent with the present model, as it predicts for a magnetized disk-corona a predominantly soft X-ray spectrum during nearly critical accretion.

Since observations indicate that both heating and acceleration of the plasma to relativistic velocities occur, as suggested by the observed disk X-ray luminosity of the order of $\sim 10^{39} \mathrm{erg} \mathrm{s}^{-1}$, and by the radio flares which in turn suggest a jet mechanical power also of the order of $\sim 10^{39} \mathrm{erg} \mathrm{s}^{-1}$ at least in the nuclear region (see, e.g, Falcke \& Biermann 1999), we will assume here that a substantial fraction of $\dot{W}_{B}$ (Eq. (12)) goes to accelerate the particles.

Let us now try to answer the second part of the question above, i.e., what could be the acceleration mechanism in the reconnection region able to produce the relativistic ejecta and the radio flare?

\section{The acceleration mechanism during the flare state}

As stressed by de Gouveia Dal Pino \& Lazarian (2001), the particular mechanism of particle acceleration during reconnection events is still unclear in spite of numerous attempts to solve the problem (see LaRosa et al 1996; Litvinenko 1996). Cosmic rays from the Sun confirm that the process is sufficiently efficient in spite of the apparent theoretical difficulties for its explanation.

Particles could, in principle, be accelerated at once to the relativistic energies by the large induced electric field within the reconnection region (e.g., Bruhwiller \& Zweibel 1992, Haswell et al. 1992; Litvinenko 1996; de Gouveia Dal Pino \& Lazarian 2000, 2001), but one-shot acceleration mechanisms can make it difficult to explain how particles will afterwards produce a power-law particle and synchrotron spectrum. Another possibility is that particles are accelerated by a Fermi process in the reconnection site. In this regard, we discuss below two viable processes.

A schematic representation of a reconnection region is shown in Fig. 3. The upper and lower parts of the magnetic flux move towards each other with the velocity $v_{\text {rec }}$. As a result, charged particles rays in the upper part of the reconnection zone "see" the lower part of the magnetic flux to approach them with a velocity $2 v_{\text {rec }}$. In this case an acceleration process analogous to the first-order Fermi acceleration of cosmic rays in magnetized shocks may take place (e.g., Longair 1992) and we can write

$\frac{\Delta E}{E}=\frac{2 v_{\text {rec }}}{c} \cos \theta$.

For a particle probability distribution $p(\theta)=2 \sin \theta \cos \theta \mathrm{d} \theta$, the average energy gain per crossing of the reconnection region is ${ }^{3}$ $\left\langle\frac{\Delta E}{E}\right\rangle=\frac{v_{\mathrm{rec}}}{c} \int_{0}^{\pi / 2} 2 \cos ^{2} \theta \sin \theta \mathrm{d} \theta=\frac{4}{3} \frac{v_{\mathrm{rec}}}{c}$

where $\theta$ is the pitch angle between the particle velocity and the magnetic field. The round trip of the particles between the upper and lower magnetic fluxes will produce an increment of energy twice as large. Thus the ratio of the particle energy, $E$, after such a round trip to its original energy, $E_{0}$, will be

$\delta=\frac{E}{E_{0}}=1+\frac{8}{3} \frac{v_{\mathrm{rec}}}{c}$

3 We note that since the particle Larmour radius around the magnetic field in the reconnection zone, $r_{B}=\gamma m_{\mathrm{e}} c^{2} /\left(\mathrm{Ze} \mathrm{B}_{\mathrm{X}}\right) \simeq 9 \times$ $10^{-6} \mathrm{~cm} \gamma_{3.5} B_{X, 7} \ll \Delta R_{\mathrm{X}}$ (where $\gamma_{3.5}=\gamma / 3.5$ is the Lorentz factor for electrons with velocities $\sim 0.92 c$ ), the particles will remain confined enough in the reconnection zone to be efficiently accelerated through the Fermi mechanism before escaping from the system. 
Now, assuming that the escape probability of a particle from the acceleration zone is similar to the one computed for a shock front, $4 v_{\text {rec }} / c$, then the probability to stay within the reconnection region is simply

$P=1-\frac{4 v_{\text {rec }}}{c}$,

and the electron particle spectrum is obtained from (see e.g., Longair 1992)

$N(E) \mathrm{d} E \sim E^{-1+\frac{\ln P}{\ln \delta}} \mathrm{d} E \propto E^{-5 / 2} \mathrm{~d} E$.

This equation indicates that the particle spectrum produced by first-order Fermi acceleration within the reconnection site is steeper than that normally produced in shocks (for which a similar simplified treatment that ignores losses and non-linearities provides $N(E) \propto E^{-2}$ ), so that the two processes may be, in principle, distinguished. Now, the synchrotron radiation from electrons with a power-law distribution $N(E) \propto E^{-p}$, has a power-law frequency radio spectrum $S_{v} \propto v^{-\alpha_{r}}$, where the spectral index is given by $\alpha_{r}=(p-1) / 2 \simeq 0.75$. Although crude, this estimated value is consistent with the observed radio spectrum during the flares $\left(\alpha_{r} \simeq 0.6\right)$.

Finally, the particle acceleration mechanism discussed above does not remove the possibility that the relativistic fluid may be also produced behind shocks. As in the Sun, plasmoids formed by reconnection of the field lines lines near the Y-zone may violently erupt and cause the formation of a shock front. This raises the alternative possibility of a first-order Fermi acceleration process of the ionized gas behind the shock front and the development of a power-law spectrum with particle spectral index of the order of two $\left(N(E) \propto E^{-2}\right)$ as remarked above and, as such, a corresponding synchrotron spectrum with a spectral index also of the order of the observed one, $S_{v} \propto v^{-0.5}$.

After a radio flare, while the system is slowly recovering its original magnetic field configuration, the plateau phase must resume. During this recovering phase, the magnetic field intensity in the X-region will be smaller, as is the mass accretion rate. In this state, the X-ray spectrum is observed to be hard and flat. As we have seen in Sect. 3.2, the model of Lin et al. (2002) predicts a harder X-ray spectrum when the accretion rate in the magnetized disk is very sub-Eddington for which a spectral index $\alpha_{r-x}<1.2$ should be expected.

\section{Conclusions and discussion}

We have investigated here the origin of the large scale superluminal ejections observed in the galactic microquasar GRS $1915+105$ during radio flare events and proposed a very simple model in which they could be due to violent magnetic reconnection episodes in the corona just above the inner edge of the magnetized accretion disk that surrounds the central $\sim 10 M_{\odot}$ black hole. In this region, when a large scale magnetic field is built by a turbulent dynamo process, and there is an approximate balance between the disk magnetic and gas+radiation pressures $(\beta \simeq 1)$, which implies a strong magnetic field intensity $B_{\mathrm{X}} \simeq 7 \times 10^{8} \mathrm{G}$, reconnection between the magnetic lines that arise from the disk with those of the magnetosphere of the BH may become violent. During this regime, the vertical magnetic flux generates a wind that will substantially remove angular momentum from the disk therefore increasing the disk mass accretion to a value near the critical one (with $\dot{M} \sim 10^{19} \mathrm{~g} \mathrm{~s}^{-1}$ ).

Whenever the system reaches the condition above and the magnetic field lines of the $\mathrm{BH}$ magnetosphere and those of the disk have opposite polarization, it may undergo an episode of violent reconnection that lasts for a very short time $\sim 3 \times$ $10^{-4} \mathrm{~s}$. A substantial part of the total magnetic energy released by reconnection $\left(\sim 2 \times 10^{39} \mathrm{erg} \mathrm{s}^{-1}\right)$ heats the coronal gas to $T_{\mathrm{c}} \simeq 5 \times 10^{8} \mathrm{~K}$ that produces a steep X-ray spectrum with the expected luminosity from observations. Assuming that the remaining released magnetic energy goes to accelerate the particles to relativistic velocities $\left(v \sim v_{\mathrm{A}} \sim c\right.$, where $v_{\mathrm{A}}$ is the Alfvén speed) in the reconnection site through first-order Fermi processes, we have found that a power-law electron distribution $N(E) \propto E^{-5 / 2,-2}$, and a synchrotron radio power-law spectrum $\left(S_{v} \propto v^{-0.75,-0.5}\right)$ are produced with radio spectral indices which are comparable to the one observed during the flares.

After each violent reconnection event, the system must resume a sub-critical disk mass accretion with smaller magnetic field intensity and a slower reconnection rate, thus recovering the more quiescent plateau state, as described in Sects. 2 to 4 .

Although until the present, GRS $1915+105$ has been the only system among the galactic accreting black holes where large scale ( $\gtrsim 100 \mathrm{AU})$ superluminal ejections have been observed (maybe because this system is the only one that meets the necessary conditions as investigated here), the magnetizeddisk-corona model discussed here could be applicable, in principle, to other X-ray binary sources, or even to the microquasar extragalactic counterparts, i.e., quasars with evidence of superluminal ejection.

Acknowledgements. This paper has benefited from many valuable comments from an anonymous referee. E.M.G.D.P. has been partially supported by grants of the Brazilian Agencies FAPESP (grant 1997/13084-3) and CNPq.

\section{References}

Arons, J. 1986, in Plasma Penetration into Magnetospheres, ed. N. Kylafis, J. Papamastorakis, \& J. Ventura (Iraklion: Crete Univ. Press), 115

Arons, J. 1993, ApJ, 408, 160

Belloni, T., Méndez, M., King, A. R., van den Klis, M., \& van Paradus, J. 1997a, ApJ, 479, L145

Belloni, T., Méndez, M., King, A. R., van den Klis, M., \& van Paradus, J. 1997b, ApJ, 488, L109

Biskamp, D., Schwarz, E., \& Drake, J. F. 1997, Phys. Plasmas, 4, 1002

Biskamp, D. 1997, in Advanced Topics in Astrophysical and Space Plasmas, ed. E. M. de Gouveia Dal Pino, A. Perat, G.A. Medina Tanco, \& A. C. L. Chian (The Netherlands: Kluwer), 165

Blandford, R. D. 1999, in Astrophysical Discs, ed. J. A. Selwood \& J. Goodman (San Francisco: ASP), ASP Conf. Ser., 160, 265

Blandford, R. D., \& Begelman, M. C. 1999, MNRAS, 303, L1

Blandford, R. D., \& Znajek, R. L. 1977, MNRAS, 179, 433

Bruhwiller, \& Zweibel 1992

de Gouveia Dal Pino, E. M., \& Lazarian, A. 2000, ApJ, 536, L31

de Gouveia Dal Pino, E. M., \& Lazarian, A. 2001, ApJ, 560, 358 
de Gouveia Dal Pino, E. M., \& Lazarian, A. 2004, in Proc. X Marcel Grossman Conference, in press

Dere, K. P. 1996, ApJ, 472, 864

Dhawan, V., Mirabel, I. F., \& Rodrig ez, L. F. 2000, ApJ, 543, 373

Falcke, H., \& Biermann, P. L. 1999, A\&A, 342, 49

Fender, R. P., Garrington, S. T., McKay, D. J., et al. 1999, MNRAS, 304,865

Fender, R. P., Rayner, B., Trushkin, S. A., et al. 2002, MNRAS, 330, 212

Frank, J., King, A., \& Derek, R. 1992, in Accretion Power in Astrophysics (Cambridge: Cambridge Univ. Press)

Gosh, P., \& Lamb, F. K. 1978, ApJ, 223, L83

Haswell, C. A., Tajima, T., \& Sakai, J.-L. 1992, ApJ, 401, 495

Jing, J., Qiu, J., Lin, J., et al. 2005, ApJ, 620, 1085

King, A. R., Pringle, J. E., West, R. G., \& Livio, M. 2004, MNRAS, 348,111

Kudoh, T., Matsumoto, R., \& Shibata, K. 2002, PASJ, 54, 267

LaRosa, T. N., Moore, R. L., Miller, J. A., \& Shore, S. N. 1996, ApJ, 467,454

Lazarian, A. 2005, in Magnetic Fields in the Universe: from Laboratory and Stars to Primordial Structures, American Institute of Physics Procs. NY, ed. E.M. de Gouveia Dal Pino, G. Lugones and A. Lazarian, in press

Lazarian, A., \& Vishniac, E. 1999, ApJ, 517, 700

Lazarian, A., \& Vishniac, E. 2000, Rev. Mex. Astron. Astrofis., 9, 55

Lazarian, A. Vishniac, E., \& Cho, J. 2004, ApJ, 603, 180

Litvinenko, Y. E. 1996, ApJ, 462, 997
Liu, B. F., Mineshige, S., \& Shibata, K. 2002, ApJ, 572, L173

Livio, M. 1997, in Accretion Phenomena and Related Outflows, ed. D.T. Wickramasinghe, G.V. Bicknell, \& L. Ferrario, ASP Conf. Ser. 121 (San Francisco: ASP), IAU Colloq. 163, 845

Livio, M., Pringle, J. E., \& King, A. R. 2003, ApJ, 593, 184

MacDonald, D. A., \& Thorne, K. S. 1982, MNRAS, 198, 345

MacDonald, D. A., Thorne, K. S., Price, R. H., \& Zhang, X.-H. 1986, in Black Holes: The Membrane Paradigm, 120

Mirabel, I. F., \& Rodriguez, L. F. 1994, Nature, 371, 46

Merloni, A. 2003, MNRAS, 341, 1051

Mirabel, I. F., \& Rodriguez, L. F. 1999, ARA\&A, 37, 409

Narayan, R., Mahadevan, R., \& Quataert, E. 1998, in The Theory of Black Hole Accretion Disks, ed. M. A. Abramowicks, G. Bjornsson, \& Pringle, J. E. (Cambridge: Cambridge Univ. Press), 148

Qiu, J., Wang, H., Cheng, C. Z., \& Gary, D. E. 2004, ApJ, 604, 900

Reames, D. V. 1995, Rev. Geophys., 33, Suppl., 585

Sakajiri, T., Brooks, D. H., Yamamoto, T., et al. 2004, ApJ, 616, 578

Shakura, N. I., \& Sunyaev, R. A. 1973, A\&A, 24, 337

Shibata, K., \& Aoki, S. 2003 [arXiv: astro-ph/0303253]

Tagger, M., Varniére, P., Rodriguez, J., et al. 2004, ApJ, 607, 410

Tagger, M., \& Pellat, R. 1999, A\&A, 349, 1003

Takasaki, H., Asai A., Kiyohara, J., et al. 2004, ApJ, 613, 592

Vishniac, E., \& Lazarian, A. 1998, ApJ, 511, 193

Wang, D. X., Xiao, K., \& Lei, W. H. 2002, MNRAS, 335, 655

Wang, D. X., \& Lei, W. H., \& Ma, R.-Y. 2003, MNRAS, 342, 851 